\title{
Comparision of BER's of QAM and PSK Modulation Techniques for Channel Estimation by using LS Estimator in MIMO-OFDM System
}

\author{
Diksha Chauhan ${ }^{1}$, Vivek Kanwar ${ }^{2}$ \\ Student, Electronics and Communication, Shoolini University, Solan, India ${ }^{1}$ \\ Assistant Professor, Electronics and Communication, Shoolini University, Solan, India ${ }^{2}$
}

\begin{abstract}
This paper gives the comparison of the Bit error rates of modulation techniques like QAM and PSK for their different order of modulations (M) and for different values of subcarriers $(\mathrm{N})$ by using LS channelestimator. Comparison of bit error rates of various modulation schemes is done to find out the best suited modulation technique for transmitting N no. of subcarriers. LS estimator is a technique used for channel estimation in MIMO-OFDM system. Channel estimation is done at the receiver for compensating the effect of distortions which are created by the channel. LS channel estimator comes under the block type channel estimation technique.
\end{abstract}

Keywords: channel estimation; modulation schemes; MIMO; OFDM; MIMO-OFDM; LS estimator; QAM; PSK.

\section{INTRODUCTION}

In communication system data and signals are transferred from one place to another by many techniques. There are two methods used for sending information or data from 1 place to another place, one is Wired technology and another one is Wireless technology. In wired technology, the information is transferred through a wired medium but in case of wireless technology the information is transferred from one place to another through wireless medium such as air.

Wireless technology is preferred over wired technology. For getting greater data rates and lower BER's we need wireless communication. They are affected by multipath propagation, so the signal propagating through them experience fading and interference [1] They give us degraded transmitted signal. Due to rapid growth in the wireless approaches we are successful to achieve very larger data rates in smaller bandwidths.

One of the technology which is used for gettinglarger data rates is known as MIMO. Now a day's telecom industry is using MIMO technology with the combination of OFDM technology. Combination of these two technologies give birth to MIMO-OFDM. It gives us good strength of signal and greater spectrum efficiency.The important challenge is to reconstruct the original signal from the received signal, for estimating channel parameters from received signal channel estimation is used [2].

For estimating the channel many modulation techniques like 2QAM, 4QAM, 8QAM, 16QAM, 2PSK, 4PSK, 8PSK, 16PSK are used with channel estimators. Pilot based channel estimation is performed by using LS and MMSE estimator [17]

\section{MIMO}

MIMO stands for Multiple Input Multiple Output. To increase the bandwidth MIMO uses multiple antennas at its receiver and transmitter end [5]. By the use of various antennas there is an increase in reliability of system because of this, wireless receiver and transmitter provides an approximately promising answer to raise the rate of the bandwidth efficiency and system security without using any supplementary bandwidth or transmitting greater power towards the channel [3]. By using spatial multiplexing greater spectral efficiency is attained. Improvement in quality of signal and the coverage range is attained by spatial diversity. MIMO capacity can be increased by using these methods:

i) Using spatial diversity power efficiency can be enhanced.

ii) By using layered method, throughput of MIMO system is enhanced.

By first method at receiver and transmitter the quality of transmission is improved. This transmission quality is described in the form of bit error rate (BER). Spatial multiplexing is used to expand the limit of MIMO. Various autonomous data streams are sent over same frequency bands in identical time slots and simultaneously from every transmitter antenna. At the receiver end these all data streams are disconnected [4]. By using MIMO technology many problems like fading and multipath are solved.In MIMO technology some transmitted signals take direct path from transmitter to receiver, whereas some signals take indirect paths to arrive the receiver. These indirect paths include paths like ground, bouncing off objects or through atmospheric layers. These all signals which are taking indirect paths to arrive the receiver usually gets attenuated. These signals reach at the receiver 
with a delay. These signals generally get ignored, but this results in lots of energy wastage. Those multipath signals which are strongest among all are difficult to ignore and they can also damage the performance of the wireless device. The strongest multipath signals are received every time and they contribute dissimilar types of delays to the received signals, troughs and peaks are forced back to alignment by them, this concept is used in MIMO and it is called that MIMO takes benefit of multipath. When different signals reach at receiver at various times then it gives birth to multipath. MIMO uses multiple spatial paths to diminish the affectability of fading. Spectral efficiency is the total number of data bits per second per $\mathrm{Hz}$ transmitted from one range to another [6].

For increasing the spectral efficiency of the MIMO system, the total amount of power that is transmitted from the transmitter, is divided into multiple paths, because of these multiple paths the capacity becomes closer to linear system for each mode and thus it gives us the increase in the spectral efficiency.

\section{OFDM SYSTEM}

In wireless systems OFDM is very popular and useful technique because it meets the requirements of future technologies.OFDM stands for Orthogonal Frequency Division Multiplexing. It is a multicarrier modulation scheme used in $4 \mathrm{~g}$ technologies [7]. It does not have very complex equalizations and it also removes ISI[8]. In OFDM firstly the signal bandwidth is devided into parallel independent data streams and then modulation is performed, this process is called multicarrier modulation. After that the signals are re-multiplexed for creating OFDM carrier. Subcarriers of ofdm are orthogonal and overlapping so because of orthogonality of subcarriers it is called OFDM. In OFDM system large no. of tightly spaced narrowband carriers are transmitted and there is no crosstalk between these subcarriers.OFDM handles multipath interference in receiver. OFDM overcomes the problems caused by multipath i.e. frequency selective fading and ISI.Because of the flatness of narrowband channel, frequency selective fading is reduced. The symbols becomes longer than the channel impulse response when they are modulated at very low rate,this cause to reduce ISI [4]. ISI can also be eliminated if guard interval is added, but it leads us to power loss and increased bandwidth of the system[10]. A drawback of OFDM is that $\mathrm{N}$ modulators and filters at transmiter and $\mathrm{N}$ demodulators and filters at receiver end make the system very complex, this complexity can be overcome by using FFT and IFT at receiver and transmitter section [9]. Generally OFDM is a combination of $\mathrm{N}$ modulated signals, in few cases some OFDM signals are in phase with each other and hence they add up and provides a large output, whereas in some cases OFDM signal components are out of phase and hence get cancelled producing zero output. For avoiding the clipping of transmitted wave, power amplifiers which have a wide linear range(for including peaks in transmitted waves) are used. This consumes very large amount of power and makes the system very costly. To maintain orthogonality between subcarriers OFDM needs synchronization between time and frequency.OFDM is very responsive to the frequency offset problem,this problem is caused by doppler shift(due to relative motion between transmitter and receiver). FFT and IFFT algorithms are used[3].OFDM have $\mathrm{N}$ overlapping (which are also orthogonal) sub bands,each of them have a baud rate of $1=t$ and have the spacing of $1=t$ [11].OFDM is mostly used because it eliminates the effect of ISI and also work under multipath by eliminating it.

\section{MIMO-OFDM SYSTEM}

For acomplishing the demands of high data rates in the limited frequency spectrum MIMO-OFDM technique is adopted. All the wireless technologies likes 4G, 3GPP LTE,Wi-max are using MIMO-OFDM nowadays for providing better signal strength,lowest bit error rate,higher signal to noise ratio and for better channel capacity and higher speed.It is a mixture of MIMO and OFDM system. The method of transmitting OFDM signals with the help of multiple antennas by using MIMO system is called MIMO-OFDM System. By using these two techniques in combination there is a improvement in data rates and channel capacity, link reliability is also improved because it fight against frequency selective fading [12]. MIMOOFDM system is devided into two parts. One is transmitter and another is receiver part. Transmitter part of MIMO-OFDM have many parallel paths.Every branch of transmitter converts serial data into parallel form, after that pilot insertion is performed for every symbol.Then by using IFFT the signal is converted from frequency domain to time domain. At the end of transmitter section cyclic prefix(the copy of last part of the OFDM symbol is added into the front of the symbol) is added to the signal.Before the transmission of signal through antennas the signal is space time encoded by making the use of Almounti algorithm. The signal is then transmitted through various $\mathrm{rf}$ antennas. The receiver section performs the opposite task of the transmitter. The cyclic prefix is removed at the receiver before the process of demodulation takes place and FFT is performed to convert the signal back from time domain to frequency domain.

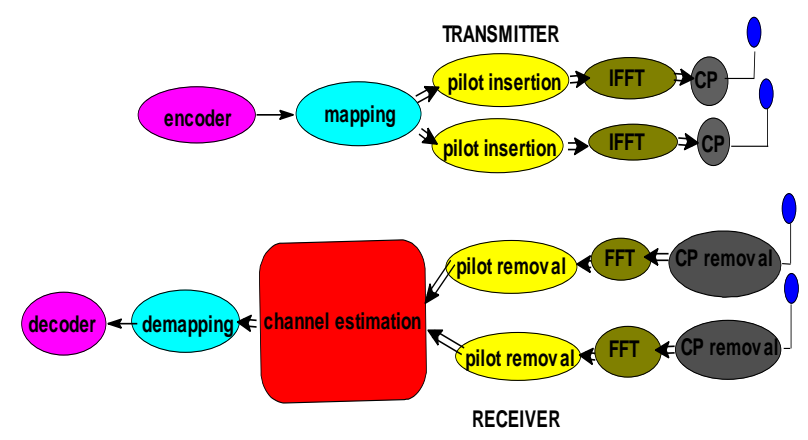

Fig.1. MIMO-OFDM system 
Transmitted symbols are combined by using space time that the reciever get the prior knowledge of the pilot decoding(parallel data is again converted in serial form). symbols which are transmitted by the transmitter. Pilot Channel estimation io also performed at the receiver end symbols are added in the transmitter[14]. There are two to recover the transmitted bits. This whole process is done methods to insert pilot symbols in the data symbol:

for achieving the required output.

\section{CHANNEL ESTIMATION}

In OFDM system the signal is transmitted from transmitter to receiver through the wireless channel, then because ofchannel characteristics the received signal is in distorted form, so to get back the transmitted information without distortion, channel effect must be estimated at the receiver. Channel estimation is done by inserting pilot bits (preamble) into the data or information, which is known to transmitter and receiver [2].

As channel capacity of MIMO-OFDM system increases linearly with the no. of antennas, this gives good results only when the receiver have the awareness about the channel state information of transmitter, but it is never possible to have the prior awareness about CSI at the receiver before the transmission. Because of this reason correct estimation is very important [13]. CSI is required for Space-Time decoding in MIMO-OFDM system. The aim of channel estimation is to look forward for the required channel information. By using the channel information the transmitter handles the coding more excellently and receiver discover the signal more effectually. It is preffered to estimate the characteristics of channel which are based on pilot signals in each separate data block of OFDM because many changes occur in the single block of OFDM. When the channel is under slow fading the channel transfer function is supposed fixed inside one OFDM block, whereas the channel transfer function for prior block work transfer function of current block [11].

In MIMO-OFDM system to reduce the effect of multipath propagation there is a need of equalization at the receiver end, it requires channel state information so channel estimation is must in MIMO-OFDM system. By using pilot symbols or preamble the channel can be estimated by using various interpolation techniques.

There are three types of channel estimation schemes

A) Pilot based channel estimation/ training sequence based channel estimation

B) Blind approach

C) Semi blind

This paper is focused on block type pilot based channel estimation technique in which performance of LS estimator is evaluated by using various modulation techniques for the purpose of channel estimation.

A) Pilot based channel estimation

In this type of channel estimation scheme pilot symbols, which are known to the receiver are combined with OFDM data to perform channel estimation. This is done to perfrorm channel estimation by the receiver, so i) Block type
ii) $\quad$ Comb type

i) Block Type:

Pilot symbols are added in each subcarrier of OFDM symbol after a periodic interval of time [1]. These pilot bitsare used as reference signals for the estimation of channel by receiver and transmitter. This method is used when channel is under slow fading and channel remains constant over some OFDM symbols [15]. In block type method time domain interpolation is achieved for estimating the channel all along time axis [2]. The response of channel is estimated by the use of LS or by using MMSE method. Fig.2 shows arrangement of OFDM symbols in block type arrangement.

ii)Comb Type:

In comb type method of pilot insertion the pilot bits are transmitted continuously (all time) but there is an even spacing on the OFDM subcarriers. And the channel is not stationary, it changes between neighboring OFDM symbols . Comb type arrangement is used when channel is under the influence of fast fading. The channel is estimated by doing the use of interpolation techniques like pecewise constant interpolation,cubic spline interpolation,line domain interpolation, $2^{\text {nd }}$ order interpolation [15] .

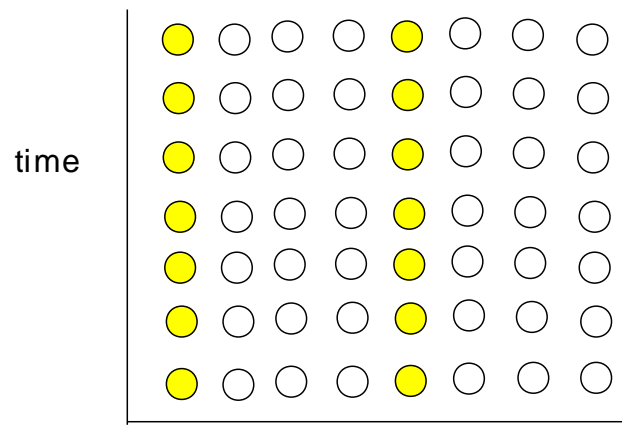

frequency

Fig.2. Block type arrangement of OFDM symbols

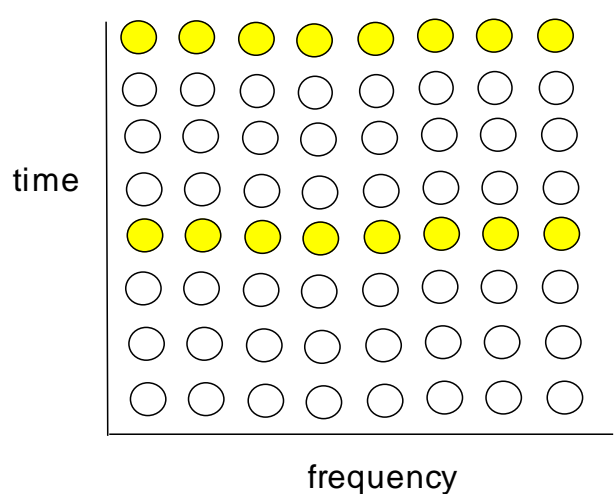

Fig.3. Comb type arrangement of OFDM symbols 
A.1Channel estimation techniques using block type arrangement

i) Least square algorithm:

LS method performs channel estimation by reducing the difference between true and actual value of the parameters of channel. By using estimated channel conditions receiver decodes the data till the arrival of next pilot symbol [16]. LS algorithm is used for estimating a system $\mathrm{h}(\mathrm{m})$ by reducing the squared error between the estimated and detected signal.

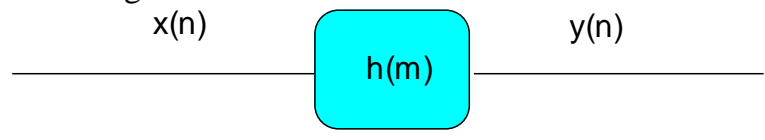

Figure 4

It is written as $\mathrm{Y}=\mathrm{XH}$

where $\mathrm{y}=$ output signal

$\mathrm{x}=$ input signal

$\mathrm{h}=$ channel

then, the error e can be written as

$\mathrm{e}=\mathrm{y}$ ' $-\mathrm{y}$

where $y=$ actual output

y'= expected output

the squared error (s) is defined by

$\mathrm{s}=|\mathrm{e}|^{2}(3)$

$\mathrm{s}=\left(\mathrm{y}^{\prime \prime}-\mathrm{y}\right)^{2}$

(4)

$\mathrm{s}=\left(\mathrm{y}^{\prime \prime}-\mathrm{y}\right) *\left(\mathrm{y}^{\prime \prime}-\mathrm{y}\right)^{\mathrm{t}}$ where $\mathrm{t}$ is complex transpose of a matrix

$\mathrm{s}=\left(\mathrm{y}^{\prime \prime}-\mathrm{xh}\right) *\left(\mathrm{y}{ }^{\prime \prime}-\mathrm{xh}\right)^{\mathrm{t}}$

then by taking its derivative w.r.t. h, we get

$\mathrm{h}^{\prime \prime}=\left(\mathrm{x}^{\mathrm{t}} \mathrm{x}\right)^{-1} \mathrm{x}^{\mathrm{t}} \mathrm{y}$

it can be written as

$h^{\prime \prime}=x^{-1} y$

hls $=x^{-1} y$

hls $=y / x$

\section{MODULATION TECHNIQUES}

Modulation techniques like QAM and PSK are used for transmitting $\mathrm{N}$ no. of subcarriers. In QAM its amplitude changes with the phase. QAM is the combination of PSK and ASK [17]. 2, 4,8and 16QAM are the different parts of QAM. 8QAM transmits 3bits per symbol,16 QAM transmit 4bits per symbol. In PSK modulation only phase changes whereas frequency and amplitude remain constant. 2PSK, which is also known as BPSK encrypts one bit per symbol whereas QPSK(4PSK) transmit 2 bits per symbol due to its 4 phases.8PSK have more than eight phases and it transmit 3 bits per symbol.16 PSK transmits 4 bits per symbol.

\section{RESULTS AND DISCUSSIONS}

The simulations are performed by using MATLAB 7.8.0(R2009a). By using LS channel estimator, modulation techniques like QAM and PSK are compared for different values of $\mathrm{M}=2,4,8,16$ and also for different values of $\mathrm{N}=64,128,256$ and 512 .
TABLE I PARAMETERS

\begin{tabular}{|l|l|}
\hline No. of subcarriers(N) & $64,128,256,512$ \\
\hline No. of pilots (P) & $8,16,32,64$ \\
\hline $\begin{array}{l}\text { Total no. of data } \\
\text { subchannels (S) }\end{array}$ & $56,112,224,448$ \\
\hline $\begin{array}{l}\text { Guard interval length } \\
\text { (GI) }\end{array}$ & $16,32,64,128$ \\
\hline Modulation (M) & 2PSK,4PSK,8PSK,16PSK \\
\hline Pilot interval (PI) & 8 \\
\hline Channel length (L) & 16 \\
\hline $\begin{array}{l}\text { No. of iteration in each } \\
\text { interval (NI) }\end{array}$ & 1000 \\
\hline
\end{tabular}

In this case no. of subcarriers are 64 , no. of pilot symbol is 8 , total no. of data subchannels are 56 and guard interval length is 16. In case of $\mathrm{N}=64$, by using PSK modulation,2PSK gives the best performance among 4PSK,8PSK and 16PSK.

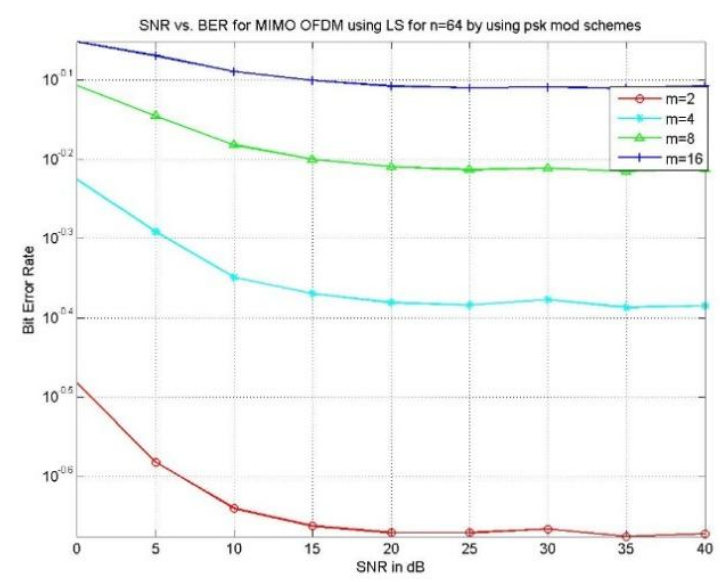

Fig. 5. SNR vs BER for $\mathrm{N}=64$ at different values of $\mathrm{M}=2,4,8,16$

TABLE II FOR N=64

\begin{tabular}{|l|l|l|l|l|}
\hline SNR & M=2PSK & M=4PSK & M=8PSK & M=16PSK \\
\hline 0 & 0.3235 & 0.5754 & 0.7762 & 0.8912 \\
\hline 5 & 0.2630 & 0.5128 & 0.7413 & 0.8511 \\
\hline 10 & 0.2238 & 0.4570 & 0.6622 & 0.8165 \\
\hline 15 & 0.2187 & 0.4365 & 0.6456 & 0.7961 \\
\hline 20 & 0.2089 & 0.4265 & 0.6309 & 0.7943 \\
\hline 25 & 0.2137 & 0.4187 & 0.6025 & 0.7603 \\
\hline 30 & 0.2041 & 0.4168 & 0.6309 & 0.7943 \\
\hline 35 & 0.2089 & 0.4187 & 0.6025 & 0.7762 \\
\hline 40 & 0.2046 & 0.4168 & 0.6025 & 0.7762 \\
\hline
\end{tabular}

Fig. 5 shows that as SNR increase BER starts decreasing, BPSK gives us less no. of errors whereas 16PSK gives worst performance in case of $\mathrm{N}=64$. Value of BER is larger in case of $M=16$. At $S N R=40$ the $B E R$ value is 0.7762 , which is very large as compare to others. So, for $\mathrm{N}=64$ BPSK is best suited. 
In fig. 6 different modulation schemes of PSK are compared for $\mathrm{N}=128$. In this case no. of subcarriers are 128 , no. of pilots is 16 , total no. of data subchannels are 112 and guard interval length is 32. BPSK is best suited among all because it gives less BER as the SNR increases. In this case, the second best is 4PSK and third best is 8PSK. 16PSK gives the worse performance because its $\mathrm{BER}$ is very high. At $\mathrm{SNR}=016 \mathrm{PSK}$ gives the value of $\mathrm{BER}=0.6456$ which is very large as compare to the others. It is 0.1445 times greater than the BER of 8PSK and 0.4218 times greater than the BER of 4PSK. 16PSK gives 0.4872 times larger error than 2PSK.

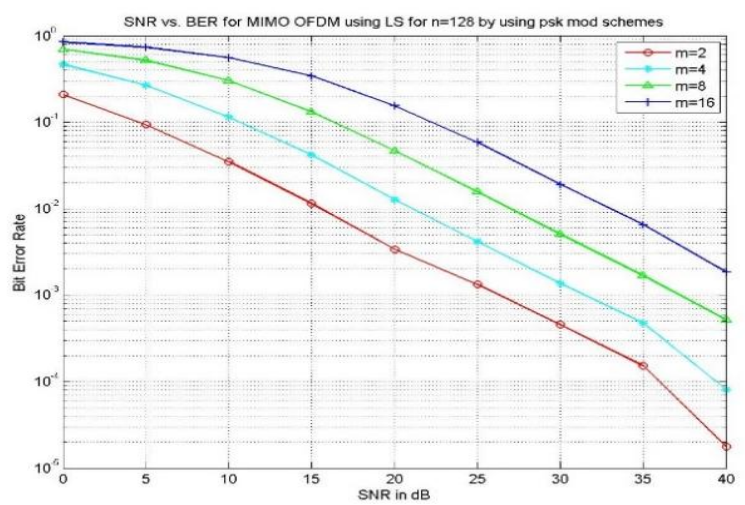

Fig. 6. BER vs. SNR graph of MIMO OFDM system by using LS channel estimator for $\mathrm{N}=128$ by using different PSK modulation schemes

TABLE III FOR N=128

\begin{tabular}{|l|l|l|l|l|}
\hline SNR & M=2PSK & M=4PSK & M=8PSK & M=16PSK \\
\hline 0 & 0.1584 & 0.2238 & 0.5011 & 0.6456 \\
\hline 5 & 0.0630 & 0.1905 & 0.3162 & 0.5011 \\
\hline 10 & 0.0177 & 0.1047 & 0.1995 & 0.3548 \\
\hline 15 & 0.0102 & 0.0199 & 0.1047 & 0.2238 \\
\hline 20 & 0.0019 & 0.0112 & 0.0251 & 0.1202 \\
\hline 25 & 0.0011 & 0.0027 & 0.0120 & 0.0316 \\
\hline 30 & 0.00019 & 0.0010 & 0.0025 & 0.0125 \\
\hline 35 & 0.000102 & 0.0002 & 0.0012 & 0.0033 \\
\hline 40 & 0.0000257 & 0.0003 & 0.0002 & 0.0012 \\
\hline
\end{tabular}

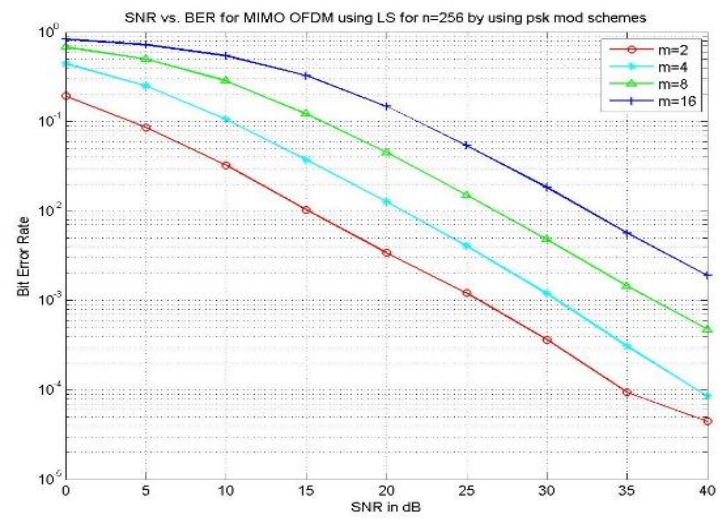

Fig. 7. SNR vs BER for MIMO OFDM systems by using

LS channel estimator for various PSK modulation schemes for $\mathrm{N}=256$
Fig.7 shows comparison of SNR vs BER for MIMO OFDM systems by using LS channel estimator for various PSK modulation schemes for $\mathrm{N}=256$. In this case no. of subcarriers are 256 , no. of pilot symbol is 32 , total no. of data subchannels are 224 and guard interval length is 64 .

\section{TABLE IV FOR N=256}

\begin{tabular}{|l|l|l|l|l|}
\hline SNR & M=2PSK & M=4PSK & M=8PSK & M=16PSK \\
\hline 0 & 0.1258 & 0.2570 & 0.4786 & 0.6456 \\
\hline 5 & 0.0562 & 0.1778 & 0.3162 & 0.5128 \\
\hline 10 & 0.0162 & 0.1 & 0.1995 & 0.3311 \\
\hline 15 & 0.0102 & 0.0199 & 0.1071 & 0.1659 \\
\hline 20 & 0.0017 & 0.0107 & 0.1 & 0.1148 \\
\hline 25 & 0.0010 & 0.0019 & 0.0223 & 0.0028 \\
\hline 30 & 0.0002 & 0.0010 & 0.0022 & 0.0120 \\
\hline 35 & 0.0001 & 0.00019 & 0.0011 & 0.0031 \\
\hline 40 & 0.0000177 & 0.000012 & 0.0002 & 0.0012 \\
\hline
\end{tabular}

Fig. 7 shows that the initial value of BER for 2PSK at $\mathrm{SNR}=0$ is 0.1258 and the last value of $\mathrm{BER}$ at $\mathrm{SNR}=40$ is 0.0000177 , which is very small and gives us the best system performance for $\mathrm{N}=256$. There is a decrease in the BER with the increase in the signal to noise ratio. Less will be the BER better will be the system performance. 16 PSK is giving the poorer performance because the value of error is large in this case, value of BER is decreasing with increase in SNR but it is not decreasing up to a desire level for better system performance as compare to the other modulation schemes, it is giving the highest BER at $\mathrm{SNR}=0$ and giving the highest value of $\mathrm{BER}$ at $\mathrm{SNR}=40$. Table III gives the values of BER for different modulation schemes for different values of SNR.

In fig. 8, PSK modulation techniques are compared for $\mathrm{N}=$ 512. SNR vs. BER curves are obtained for modulation schemes like BPSK, QPSK, 8PSK and 16PSK by using LS estimator method of channel estimation in MIMO OFDM system.2PSK is giving the better performance in case of $\mathrm{N}=512$ and 16PSK is giving the worse performance because at $\mathrm{SNR}=40$ it is giving $\mathrm{BER}=0.0012$ which is larger as compare to other modulations.

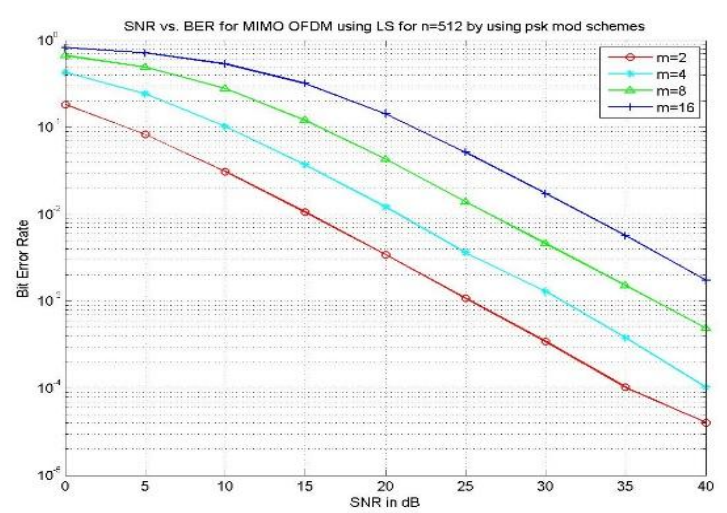

Fig. 8SNR vs BER for MIMO OFDM systems by using LS channel estimator for various PSK modulation schemes for $\mathrm{N}=512$ 
From all above graphs of PSK it is concluded that as order BER at SNR=40 is smaller for $\mathrm{m}=2 \mathrm{QAM}$ as compare to of modulation increases, BER also starts increasing. But 4QAM, 8QAM, 16QAM. The worst performance is given for better performance of system we want less value of by $M=16$ as it gives the highest BER among other BER. It is also shown that as we increase no. of modulation schemes.4QAM gives the second-best subcarriers the modulation schemes give better performance after 2QAM, its BER is 0.4120 at $\mathrm{SNR}=40$. performance, so the system gives better performance if we 2QAM is better than 4QAM by the difference of 0.2093 transmit 512 subcarriers with 2PSK scheme, it gives us and 16QAM by the difference of 0.6563 and 8QAM by the less BER of 0.000015 at $\mathrm{SNR}=40$. difference of 0.5301 .

TABLE VFOR N=512

\begin{tabular}{|l|l|l|l|l|}
\hline SNR & M=2PSK & M=4PSK & M=8PSK & M=16PSK \\
\hline 0 & 0.1230 & 0.2511 & 0.4466 & 0.6309 \\
\hline 5 & 0.0562 & 0.1380 & 0.3162 & 0.5011 \\
\hline 10 & 0.0158 & 0.1 & 0.1949 & 0.3311 \\
\hline 15 & 0.01 & 0.0190 & 0.1071 & 0.2041 \\
\hline 20 & 0.0017 & 0.0104 & 0.0204 & 0.1122 \\
\hline 25 & 0.0010 & 0.0019 & 0.0112 & 0.0257 \\
\hline 30 & 0.0001 & 0.0010 & 0.0022 & 0.0120 \\
\hline 35 & 0.000063 & 0.00019 & 0.0011 & 0.0031 \\
\hline 40 & 0.000015 & 0.00010 & 0.0002 & 0.0012 \\
\hline
\end{tabular}

Now the simulations are performed for Quadrature amplitude modulation

\section{TABLE VIPARAMETERS}

\begin{tabular}{|l|l|}
\hline No. of subcarriers(N) & $64,128,256,512$ \\
\hline No. of pilots (P) & $8,16,32,64$ \\
\hline $\begin{array}{l}\text { Total no. of data } \\
\text { subchannels (S) }\end{array}$ & $56,112,224,448$ \\
\hline Guard interval length (GI) & 16,32,64,128 \\
\hline Modulation (M) & $\begin{array}{l}\text { 2QAM, 4QAM, 8QAM, } \\
\text { 16QAM }\end{array}$ \\
\hline Pilot interval (PI) & 8 \\
\hline Channel length (L) & 16 \\
\hline $\begin{array}{l}\text { No. of iteration in each } \\
\text { interval (NI) }\end{array}$ & 1000 \\
\hline
\end{tabular}

Fig.9 shows that when $\mathrm{N}=64$, by using QAM modulation $\mathrm{m}=2$ is giving the best performance.

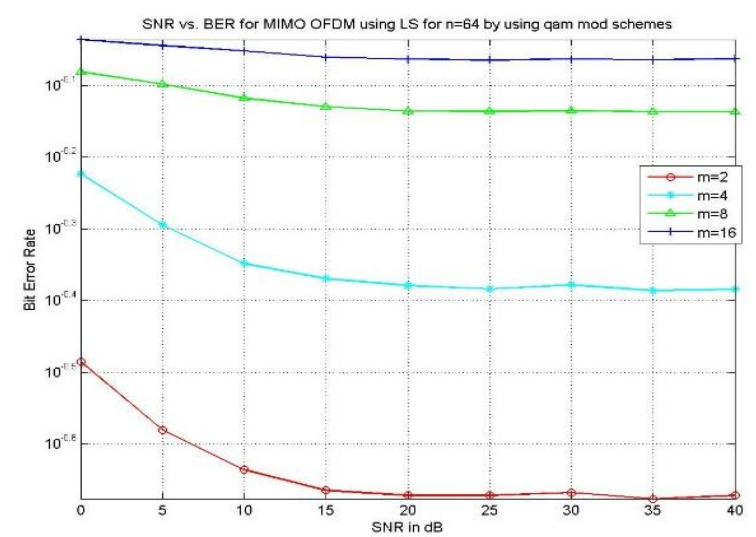

Fig.9 BER vs. SNR graph of MIMO OFDM system by using LS channel estimator for $\mathrm{N}=64$ by using different QAM modulation schemes for $\mathrm{M}=2,4,8,16$
TABLE VII FOR N=64

\begin{tabular}{|l|l|l|l|l|}
\hline SNR & M=2QAM & M=4QAM & M=8QAM & M=16QAM \\
\hline 0 & 0.3311 & 0.6309 & 0.8317 & 0.9225 \\
\hline 5 & 0.2754 & 0.5128 & 0.7943 & 0.9036 \\
\hline 10 & 0.2238 & 0.4560 & 0.7709 & 0.8851 \\
\hline 15 & 0.2089 & 0.4365 & 0.7413 & 0.8729 \\
\hline 20 & 0.2041 & 0.4168 & 0.7244 & 0.8689 \\
\hline 25 & 0.1995 & 0.4073 & 0.7227 & 0.8649 \\
\hline 30 & 0.2027 & 0.4168 & 0.7379 & 0.8669 \\
\hline 35 & 0.2027 & 0.4120 & 0.7328 & 0.8590 \\
\hline 40 & 0.2027 & 0.4120 & 0.7328 & 0.8590 \\
\hline
\end{tabular}

Fig. 10 shows the case when no. of subcarriers are 128, no. of pilots is 16 total no. of data subchannels are 112 and guard interval length is 32 .

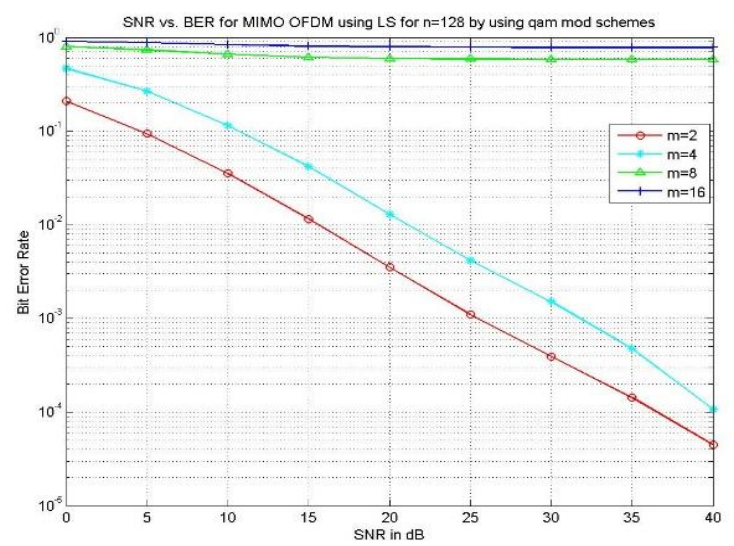

Fig.10 BER vs. SNR graph of MIMO OFDM system by using LS channel estimator for $\mathrm{N}=128$ by using different QAM modulation schemes for $\mathrm{M}=2,4,8,16$

TABLE VII FOR $\mathrm{N}=128$

\begin{tabular}{|l|l|l|l|l|}
\hline SNR & $\begin{array}{l}\text { M=2QA } \\
\text { M }\end{array}$ & $\begin{array}{l}\text { M=4QA } \\
\text { M }\end{array}$ & $\begin{array}{l}\text { M=8QA } \\
\text { M }\end{array}$ & $\begin{array}{l}\text { M=16Q } \\
\text { AM }\end{array}$ \\
\hline 0 & 0.1258 & 0.3090 & 0.7943 & 0.9120 \\
\hline 5 & 0.0794 & 0.1905 & 0.7244 & 0.7943 \\
\hline 10 & 0.0177 & 0.1047 & 0.6606 & 0.7709 \\
\hline 15 & 0.0104 & 0.0199 & 0.6025 & 0.7413 \\
\hline 20 & 0.0019 & 0.0117 & 0.5754 & 0.7244 \\
\hline 25 & 0.0010 & 0.0022 & 0.5623 & 0.7227 \\
\hline 30 & 0.0002 & 0.0010 & 0.5623 & 0.7379 \\
\hline 35 & 0.0001 & 0.0003 & 0.5508 & 0.7328 \\
\hline 40 & 0.0000251 & 0.0001 & 0.5495 & 0.7328 \\
\hline
\end{tabular}


2QAM modulation is best suited for transmitting $\mathrm{N}=128$ because 2QAM gives small BER of 0.0000251 at $\mathrm{SNR}=40.2^{\text {nd }}$ best suited modulation order for transmitting $\mathrm{N}=128$ is 4QAM because it gives the BER of 0.0001 at $\mathrm{SNR}=40$.The third best is 8QAM and the worst case is 16QAM, because 16QAM gives the higher bit error rate of 0.7328 which is very large as compare to others.8QAM and 16QAM are considered as the worst case because their values of BER is very large and the graph for both of these remains almost constant for some values of SNR and there is a very small difference of values of BER between these two modulation orders.

Fig.11 shows the BER vs. SNR graph of MIMO OFDM system by using LS channel estimator for $\mathrm{N}=256$ by using different QAM modulation schemes.In this case no. of subcarriers are 256 , no. of pilots is 32 , total no. of data subchannels are 224 and guard interval length is 64.8QAM and 16QAM are taken as poorest order modulations for transmitting $\mathrm{N}=256$ because they give the higher errors rates and BER does not decrease to required level for better performance.2QAM gives the better performance because it gives the smallest BER among all modulation orders for $\mathrm{N}=256.2 \mathrm{QAM}$ gives the BER of0.000019 at $\mathrm{SNR}=40$ and 4QAM gives the BER of 0.00012 at SNR $=40$ which is 0.00010 larger than the BER of 2QAM.

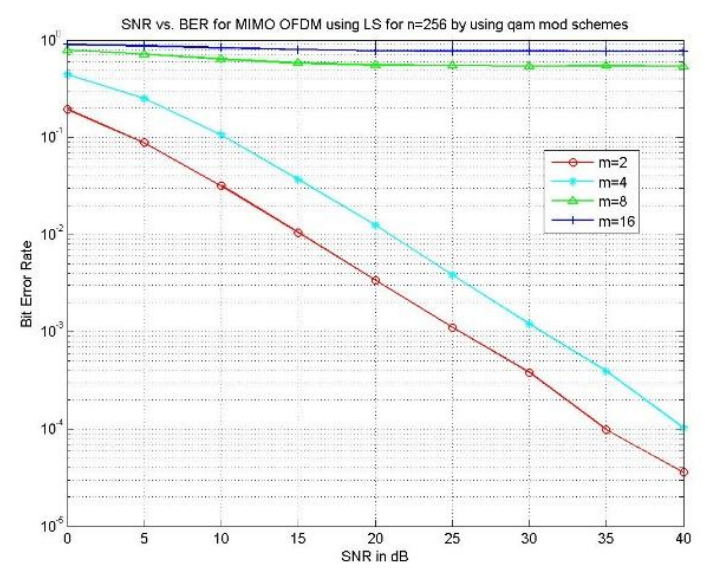

Fig.11. BER vs. SNR graph of MIMO OFDM system by using LS channel estimator for $\mathrm{N}=256$ by using different QAM modulation schemes

TABLEIXFOR N=256

\begin{tabular}{|l|l|l|l|l|}
\hline SNR & M=2QAM & M=4QAM & M=8QAM & M=16QAM \\
\hline 0 & 0.1230 & 0.2570 & 0.7585 & 0.9015 \\
\hline 5 & 0.0630 & 0.1778 & 0.7227 & 0.8709 \\
\hline 10 & 0.0162 & 0.1023 & 0.6456 & 0.8317 \\
\hline 15 & 0.01 & 0.0194 & 0.5754 & 0.7943 \\
\hline 20 & 0.0017 & 0.0107 & 0.5508 & 0.7762 \\
\hline 25 & 0.0010 & 0.0020 & 0.5495 & 0.7585 \\
\hline 30 & 0.0001 & 0.0015 & 0.5432 & 0.7585 \\
\hline 35 & 0.00005 & 0.00019 & 0.5382 & 0.7413 \\
\hline 40 & 0.000019 & 0.00012 & 0.5370 & 0.7413 \\
\hline
\end{tabular}

Fig. 12 shows the BER vs. SNR graph of MIMO OFDM system by using LS channel estimator for $\mathrm{N}=512$ by using different QAM modulation schemes.In this case no. of subcarriers are 512, no. of pilots is 64, total no. of data subchannels are 448 and guard interval length is 128.In QAM the worst case is 16QAM where $\mathrm{M}=16$ at $\mathrm{SNR}=40$ the value of BER is 0.7413 . It is giving the highest BER among 2QAM,4QAM and 8QAM.BER values of 16QAM and 8QAM are very large, the BER does not decreases linearly with increase in the SNR but it remains almost constant after SNR reaches to 30.There is a very small change in the BER's of these two modulation orders. The best case here is 2QAM, it gives the minimum value of $\mathrm{BER}$ at $\mathrm{SNR}=40$. Less the no. of errors and better will be the system performance, so 2QAM is best suited for transmitting $\mathrm{N}=512$.

It shows that with increase in modulation order the no. of errors increases, it also shows that if no. of subcarriers increases then BER decreases and system gives us good performance so 2QAM with $\mathrm{N}=512$ is best suited for LS estimators.It gives us minimum BER at high value of SNR.

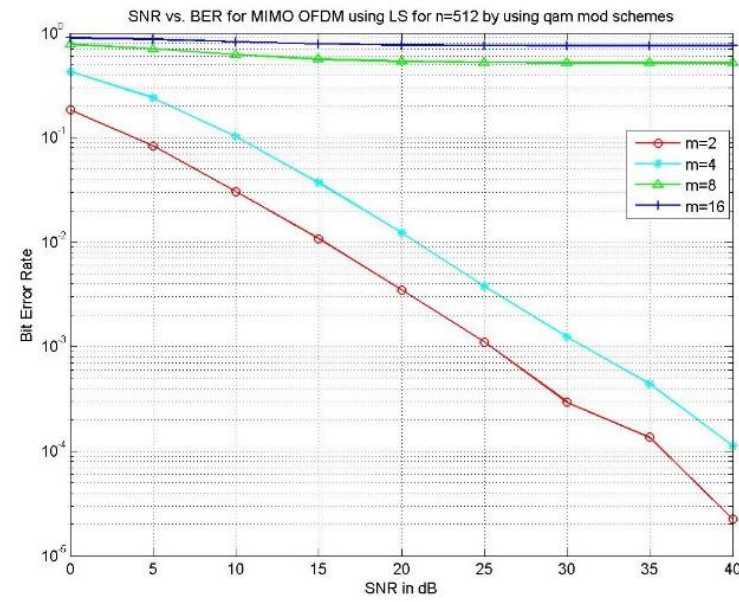

Fig.12. SNR vs BER for MIMO OFDM systems by using LS channel estimator for various QAM modulation schemes for $\mathrm{N}=512$

TABLE X FOR N=512

\begin{tabular}{|l|l|l|l|l|}
\hline SNR & M=2QAM & M=4QAM & M=8QAM & M=16QAM \\
\hline 0 & 0.9549 & 0.2570 & 0.7585 & 0.8912 \\
\hline 5 & 0.0501 & 0.1288 & 0.7079 & 0.8709 \\
\hline 10 & 0.0158 & 0.1 & 0.01 & 0.8298 \\
\hline 15 & 0.01 & 0.0194 & 0.5623 & 0.7943 \\
\hline 20 & 0.0017 & 0.0158 & 0.5370 & 0.7762 \\
\hline 25 & 0.001 & 0.0019 & 0.5248 & 0.7585 \\
\hline 30 & 0.0001 & 0.0015 & 0.5128 & 0.7585 \\
\hline 35 & 0.0001 & 0.0001 & 0.5128 & 0.7413 \\
\hline 40 & 0.00001 & 0.00001 & 0.5128 & 0.7413 \\
\hline
\end{tabular}

From above simulations, it is also concluded that $2 \mathrm{PSK}$ is better than 2QAM in case of $\mathrm{N}=512$, because $2 \mathrm{PSK}$ gives better BER as compare to 2QAM. There values are almost 
same at the starting but at the end when SNR reaches 40 $\mathrm{BER}$ of $2 \mathrm{PSK}=0.0000104$ and BER of $2 \mathrm{QAM}=0.0000194$. So 2PSK is better than 2QAM by the difference of 0.000009 in their BER's.

\section{CONCLUSION}

Least square estimator is used for channel estimation purpose in MIMO-OFDM system because of its simplicity. In LS estimator, the best suited modulation for transmittingN no. of subcarriers is BPSK modulation because it gives less no. of errors as compare to QAM. It is also concluded that PSK and QAM start performing better as no. of subcarriers increase. For less no. of subcarriers, they both gives higher bit errors and for higher no. of subcarriers they give lesser bit error rates.

\section{REFERENCES}

[1] Y. S Cho et al, "MIMO-OFDM Wireless communication with MATLAB", IEEE Press, John Wiley and Sons, 2010.

[2]. Raspinderjit Kaur Kahlon, Gurpreet Singh Walia, Anu Sheetal3, "Channel Estimation Techniques in MIMO-OFDM Systems Review Article", International Journal of Advanced Research in Computer and Communication Engineering Vol. 4, Issue 5, May 2015

[3] Mr. S. Lenin1, Dr. S. Malarkkan "An extensive review of significant researches on channel estimation in MIMO-OFDM" Journal of Theoritical and applied information technology, Vol. 64No. $2,20^{\text {th }}$ june 2014

[4] Arun Agarwal, Saurabh N. Mehta, "Design and Performance Analysis of MIMO- OFDM System Using Different Antenna configurations", International Conference on Electrical, Electronics, and Optimization Techniques (ICEEOT) - 2016

[5] Abhishek Tandon, KeshavMehrotra, RuchiVarshney "A review of mimo systems in wireless communication "International Journal of Scientific Research and Management Studies (IJSRMS) ISSN: 2349-3771 Volume 3 Issue 1, pg: 55-60

[6]. Daniel W. Bliss, Keith W. Forsythe, and Amanda M. Chan, "MIMO Wireless Communication" Lincoln Laboratory Journal Volume 15, Number 1, 2005.

[7]. Leila Sahraoui, DjmailMessadeg, NouredinneDoghmane "Analyses and performance of techniques papr reduction for stbcmimo-ofdm system in $(4 \mathrm{~g})$ wireless communication" International Journal of Wireless \& Mobile Networks (IJWMN) Vol. 5, No. 5, October 2013

[8]. Seung Won Kang and KyungHi Chang, "A Novel Channel Estimation Scheme for OFDM/OQAM-IOTA System”, ETRI Journal, Vol. 29, No. 4, pp. 430-436, Aug 2007

[9]. Deepika Sharma, Rajiv Chechi, "To Study Channel Estimation Techniques in MimoOfdm System" IOSR Journal of Electronics and Communication Engineering (IOSRJECE) ISSN: 2278-2834 Volume 2, Issue 3 (July-Aug 2012), PP 46-49

[10]. B Shoba1 and Dr.KJayanthi, "Performance improvement of MIMOOFDM systems through channel estimation", International Journal of Wireless \& Mobile Networks (IJWMN) Vol. 4, No. 5, October 2012.

[11]. Prasanta Kumar Pradhan, Oliver Fausty, Sarat Kumar Patra and BengKon Chua, "Channel Estimation Algorithms for OFDM Systems", In Proceedings of International Conference on Electronics Systems, Rourkela, 2011.

[12].H. Bolsckei "Principles of MIMO-OFDM Communication", Communications Technology Lab, Swiss Federal Inst. Of Technology, Zurich, 2006.

[13]. Y. Shen and E. Martinez, "Channel Estimation in OFDM Systems", Application Note, Freescale Semiconductors Inc., 2006.

[14]. D.B.Bhoyar,Vaishali B. Niranjane, "Channel Estimation for MIMO-OFDM Systems", International Journal of Engineering
Research and Applications (IJERA) ISSN: 2248-9622 Vol. 2, Issue 1, Jan-Feb 2012, pp.044-050

[15]. Rajbir Kaur, Charanjit Kaur, "Investigation on Channel Estimation techniques for MIMO- OFDM System for QAM/QPSK Modulation", International Journal of Computational Engineering Research Vol. 2 Issue.5

[16]. K. Vidhya, Dr.K.R.Shankar Kumar, Pilot based channel estimation for MIMO-OFDM systems, IRACST - International Journal of Computer Networks and Wireless Communications (IJCNWC), ISSN: 2250-3501 Vol.3, No2, April 2013

[17]. AvinashSahu, Dr.AnubhutiKhare, "A Comparative Analysis of LS and MMSE Channel Estimation Techniques for MIMO-OFDM System", AvinashSahu Int. Journal of Engineering Research and Applications ISSN: 2248-9622, Vol. 4, Issue 6(Version 6), June 2014, pp.162-167

[18]. Vikas Kumar Batav, BrajlataChourasiya "Channel Estimation in Mobile Wireless Systems \& Comparisons Using Different Techniques" International Journal of Advanced Research in Computer Engineering \& Technology (IJARCET) Volume 3, Issue 6 , June 2014

\section{BIOGRAPHIES}

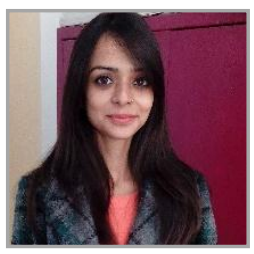

Miss. Diksha Chauhan is pursuing M.Tech in Electronics and Communication Engineering from Shoolini University Solan (H.P.). She obtained B.Tech degree in Electronics and Communication Engineering from Shoolini University Solan (H.P).She has done 3 years diploma in Electronics and Communication Engineering from Govt. Polytechnic Rohru (H.P).Her research interests are wireless communication, MIMO, OFDM

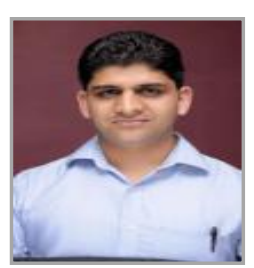

Mr. Vivek Kanwar received his M.Tech and B.Tech degree in Electronics and Communication Engineering from Jaypee University of Information Technology, Solan (H.P). Now he is currently working as Assistant Professor in Shoolini University Solan, (H.P). His current research interest areas are OFDM, MIMO, Wireless mobile communication, 4G technologies 